\title{
Liver profile in COVID-19: a meta-analysis
}

\author{
Abhishek Bansal $^{1}$ - Jang Bahadur Prasad ${ }^{2}$
}

Received: 23 April 2020 / Accepted: 7 May 2020 / Published online: 21 May 2020

(C) Springer-Verlag GmbH Germany, part of Springer Nature 2020

\begin{abstract}
Background Few studies have been performed to investigate multiorgan failure occurring with extrapulmonary symptoms like diarrhea, anorexia, and vomiting. There has been no attempt at a systematic review on this major health issue. Hence, in this study, a meta-analysis was undertaken to quantify the overall risk of liver damage in COVID-19 patients and also find the overall variation in risk outcome.

Materials and methods A meta-analysis was carried out on the findings of four published studies that were related to the albumin, alanine aminotransferase (ALT), and aspartate aminotransferase (AST) levels of COVID-19 patients. The pooled mean for each of these three measurements was estimated by using a random-effects model.

Results The pooled means for albumin, AST, and ALT in COVID-19 patients were $38.84 \mathrm{~g} / \mathrm{L}$ [95\% confidence interval (CI): 35.83-41.86], 27.28 IU/L (95\% CI: 18.30-36.26), and 24.44 IU/L (95\% CI: 15.73-33.15) respectively. There was a high degree of divergence among the studies, with the index of heterogeneity being more than $90 \%$.

Conclusion The ALT and albumin pooled means were reported to be within the normal range; however, the pooled mean of the serum AST level was found to be high in COVID-19 patients. The pooled means reported in this study can be useful for working out the degree of liver damage in COVID-19 patients.
\end{abstract}

Keywords COVID-19 $\cdot$ Liver profile $\cdot$ Albumin $\cdot$ ALT $\cdot$ AST

\section{Introduction}

An unknown outbreak of pneumonia occurred in Wuhan in December 2019 and rapidly spread all over China and, now, the whole world. This virus has some uncommon extrapulmonary symptoms in patients, like anorexia, diarrhea, and vomiting, not necessarily with respiratory symptoms (Pan et al. 2020), leading to its naming of SARS-CoV-2, with the disease being termed COVID-19. It is spread from person to person in the community. However, there is no drug on the market with definite curing effects, so the main strategy is focused on symptomatic support. Some patients showed

Abhishek Bansal

bansalabhishek99@gmail.com

Jang Bahadur Prasad

jbiips12@gmail.com

1 Department of Biochemistry, J.N. Medical College, KLE University, Belagavi 590010, India

2 Department of Epidemiology and Biostatistics, KLE University, Belagavi, India lower ability to respond to treatment after hospitalization and developed severe pneumonia, pulmonary edema, acute respiratory distress syndrome (ARDS), or multiple organ failure (Mo et al. 2020).

The first cases of the COVID-19 disease were directly linked to exposure to the Huanan Seafood Wholesale Market of Wuhan, so the animal-to-human route of transmission was presumed as the main mechanism. Transmission of the aerosol is also possible in protracted exposure. The pathophysiology of these viruses is that they are enveloped, positive-stranded RNA viruses with nucleocapsids, with an approximate length of $30 \mathrm{~kb}$, making them the largest known RNA viruses. In the host, firstly, the synthesis of polyprotein $1 \mathrm{a} / 1 \mathrm{ab}(\mathrm{pp} 1 \mathrm{a} / \mathrm{pp} 1 \mathrm{ab})$ takes place from the viral RNA. The clinical variety of COVID-19 varies from asymptomatic or paucisymptomatic forms to respiratory failure to multiorgan and systemic manifestations in terms of sepsis, septic shock, and multiple organ dysfunction syndrome (MODS). The symptoms in the early stages of infection were non-specific, and differential diagnosis may include a variety of infectious and non-infectious etiologies (Cascella et al. 2020).

Plasma albumin concentration helps in the determination of hepatocellular damage because of its synthesis in the liver and 
it has a half-life of 17 days. The normal serum level of albu$\min$ is $35-52 \mathrm{~g} / \mathrm{L}$. Aspartate aminotransferase (AST) and alanine aminotransferase (ALT) are the most important clinical aminotransferases, and the liver is a rich source. Therefore, following any damage in liver cells, these enzymes fuse into the bloodstream and increase their concentration in serum. The normal serum level of AST is 6-25 IU/L and that for ALT is 3-30 IU/L (Kaplan et al. 1995).

Some studies have noted that, in coronavirus, multiorgan failure occurs with extrapulmonary symptoms like diarrhea, anorexia, vomiting, etc. (Cascella et al. 2020; Sun et al. 2020). However, there has been no attempt at a systematic review for this major health issue. In an effort to address this gap in knowledge, this study undertook a meta-analysis to quantify the overall risk of liver damage in COVID-19 patients and also find the overall variation in risk outcome.

\section{Materials and methods}

\section{Selection of test parameters}

In the liver profile, there is a list of tests available for the determination of liver function. ALT, AST, and albumin are routinely used to test the liver profile for the determination of liver function. These are simple to perform. There are some studies in China that showed that these levels are significantly altered in COVID-19 patients (Liu et al. 2020a, b; Mo et al. 2020; Pan et al. 2020). Among these studies, some relevant studies, which were freely accessible and conducted in China among COVID-19 patients, were taken into account. They are presented in Figs. 2a, 3a, and 4a, with their separate and combined risk, analyzed by a random-effects model.

\section{Literature search}

A literature exploration was conducted using the PubMed database for locating and accessing articles related to the forms of COVID-19 associated with liver injury. The keywords used for the search were 'COVID-19', 'liver profile', and 'China'. Only full-length published articles and written in either English or Chinese were considered for this study. The references listed in these articles were also accessed for additional support.

\section{Inclusion and exclusion criteria}

Studies were considered for the meta-analysis if they pertained to associations between COVID-19 and liver profile, and the estimated liver profile was included in the metaanalysis. The following selection criteria were also applied to studies: (1) the study must have a cross-sectional or case series design and (2) the study must have a reported average estimate and their corresponding 95\% confidence intervals (CIs) of the albumin, AST, and ALT levels. A flow chart of the study selection process, along with numbers selected and excluded at different levels, is shown in Fig. 1.

\section{Data extraction}

The epidemiological guidelines of observational studies for meta-analyses were followed (Jussawalla and Deshpande 1971). From each study, the following information was extracted: (1) first author's last name, (2) year of publication, (3) number of patients, and (4) average (mean) and corresponding 95\% CIs for albumin, AST, and ALT levels separately (Table 1.

\section{Statistical analysis}

Separate contingency tables were developed for albumin, AST, and ALT. The overall mean (combined for all existing cross-sectional and case series studies) and its 95\% CI was calculated by using the random-effects model for albumin, AST, and ALT because the data used in the meta-analysis were supposed to be a random selection from all possible studies. The studies are hospital-based cross-sectional and

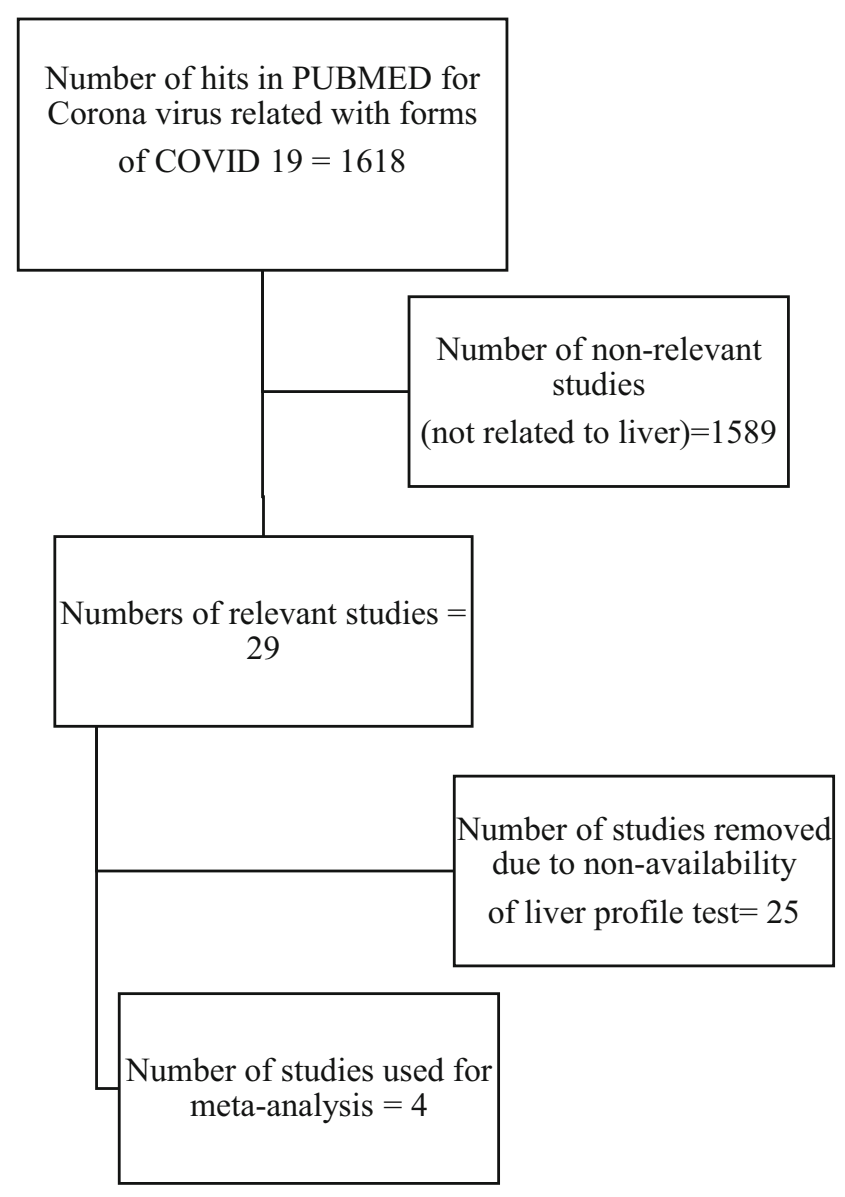

Fig. 1 Flow chart of the study selection process 
Table 1 Study characteristics

\begin{tabular}{|c|c|c|c|c|}
\hline Reference & Publication year & Place & Sample size & Mean \\
\hline Mo et al. (2020) & 2020 & Wuhan & 85 & $\begin{array}{l}\text { Albumin }=36.00 \mathrm{~g} / \mathrm{L} \\
\mathrm{AST}=37.00 \mathrm{IU} / \mathrm{L} \\
\mathrm{ALT}=28.00 \mathrm{IU} / \mathrm{L}\end{array}$ \\
\hline Liu et al. (2020b) & 2020 & Wuhan & 78 & $\begin{array}{l}\text { Albumin }=40.47 \mathrm{~g} / \mathrm{L} \\
\mathrm{AST}=20.50 \mathrm{IU} / \mathrm{L} \\
\mathrm{ALT}=18.10 \mathrm{IU} / \mathrm{L}\end{array}$ \\
\hline Liu et al. (2020a) & 2020 & Wuhan & 30 & $\begin{array}{l}\text { Albumin }=42.00 \mathrm{~g} / \mathrm{L} \\
\mathrm{AST}=20.00 \mathrm{IU} / \mathrm{L} \\
\mathrm{ALT}=18.00 \mathrm{IU} / \mathrm{L}\end{array}$ \\
\hline Pan et al. (2020) & 2019 & Wuhan & 204 & $\begin{array}{l}\text { Albumin }=36.10 \mathrm{~g} / \mathrm{L} \\
\mathrm{AST}=35.60 \mathrm{IU} / \mathrm{L} \\
\mathrm{ALT}=35.80 \mathrm{IU} / \mathrm{L}\end{array}$ \\
\hline
\end{tabular}

case series types and were carried out in China. A forest plot and funnel plot were prepared using STATA 16 statistical software to describe individual studies and the pooled mean. Heterogeneity between studies was indicated by the $\mathrm{I}^{2}$ value. $\mathrm{I}^{2}$ statistics of less than $50 \%$ were considered as low and indicating a greater degree of similarity between studies.
Statistical significance was fixed at a $p$-value less than 5\% because meta-analysis is a type of observational study; errors can occur in the methodology of study inclusion and analysis, resulting in incorrect results. The funnel graph can be used to evaluate the bias of the study, as shown in Figs. $2 b, 3 b$, and $4 b)$.
Fig. 2 a Forest plot of randomeffects meta-analysis for albumin. b Funnel plot with pseudo 95\% confidence interval for albumin

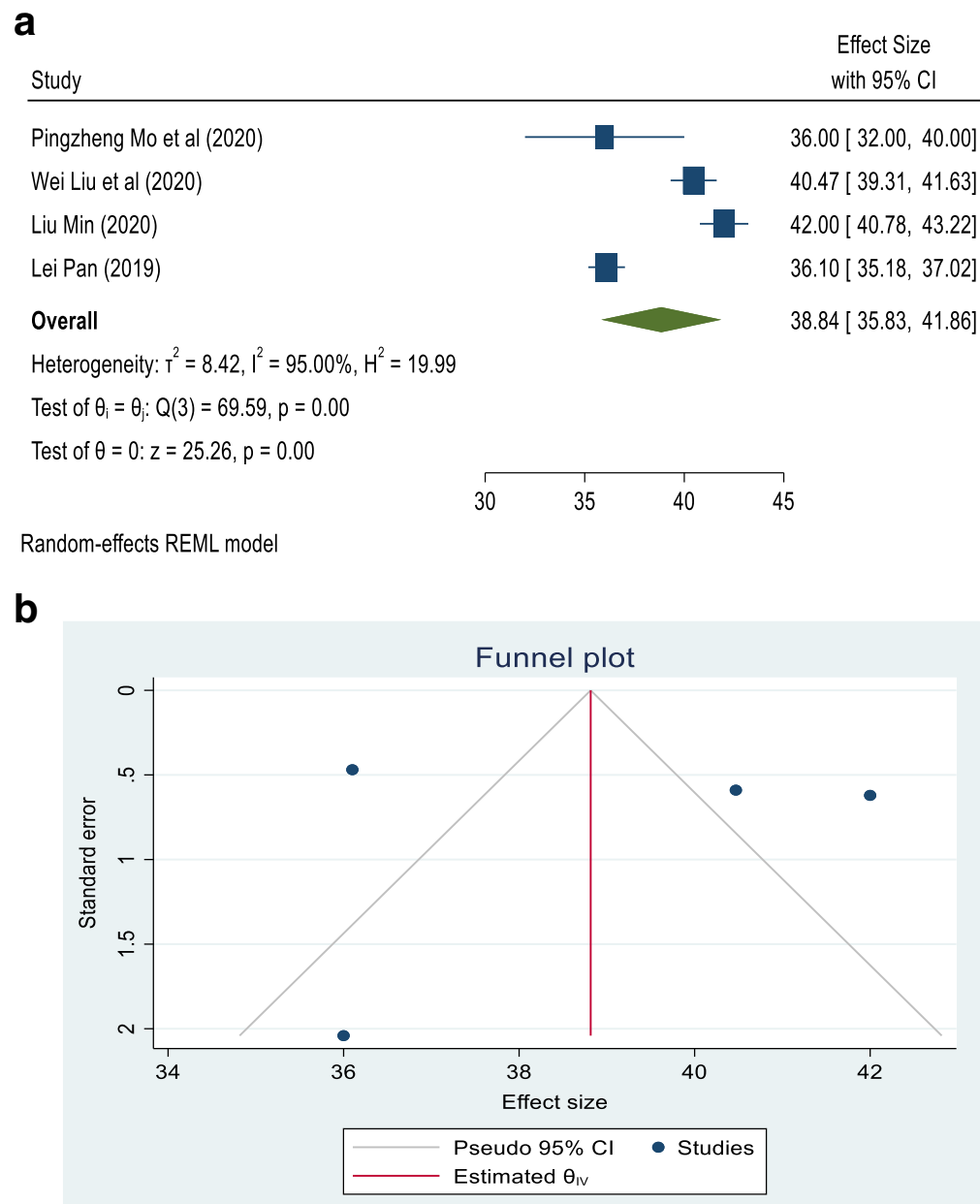


Fig. 3 a Forest plot of randomeffects meta-analysis for aspartate aminotransferase (AST). b Funnel plot with pseudo 95\% confidence interval for AST a

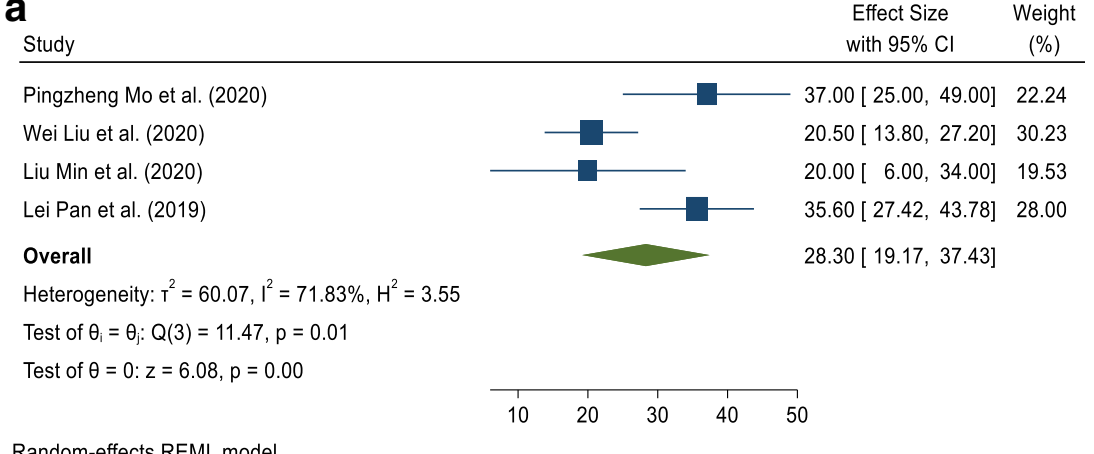

Random-effects REML model

b

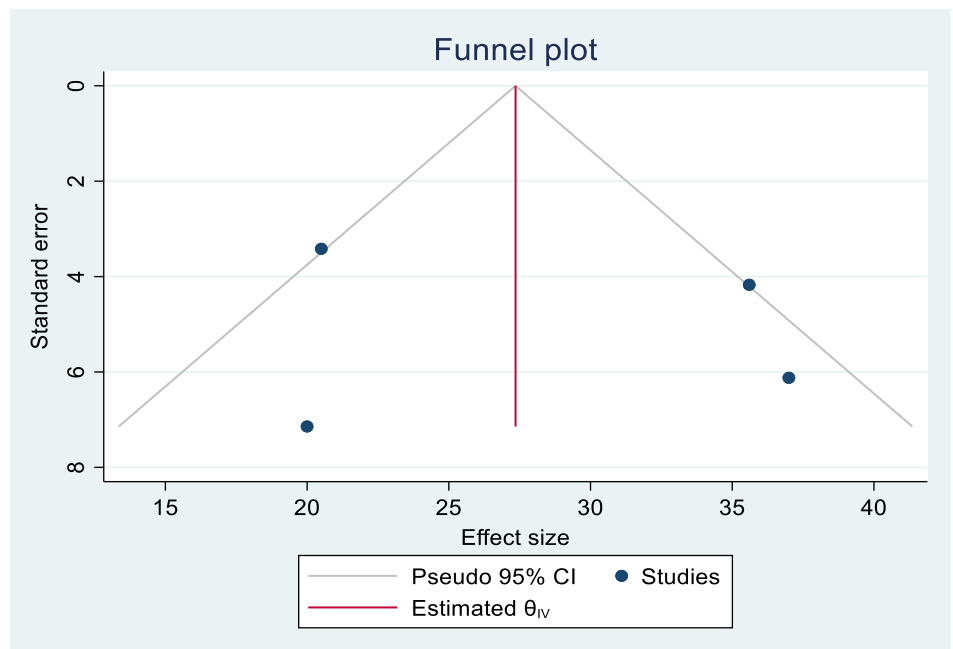

\section{Results}

Four articles that met the eligibility criteria for this study and investigated the association of COVID-19 infection with albumin, AST, and ALT levels were included in this study (Liu et al. 2020a, b; Mo et al. 2020; Pan et al. 2020). Table 2 summarizes the results of our study in the form of pooled mean, lower limit, upper limit, $\mathrm{I}^{2}$, and $p$-value of albumin, AST, and ALT associated with COVID-19. The pooled means for albumin, AST, and ALT in COVID-19 patients were $38.84 \mathrm{~g} / \mathrm{L}$ (95\% CI: 35.83-41.86), $27.28 \mathrm{IU} / \mathrm{L}$ (95\% CI: $18.30-36.26)$, and 24.44 IU/L (95\% CI: 15.73-33.15), respectively. There was a high degree of divergence among the studies, with the index of heterogeneity being more than 90\% (Table 2).

\section{Albumin}

All four studies dealing with albumin reported that its association with COVID-19 was statistically significant. The pooled mean for all four studies was 38.84 g/L (95\% CI: 35.8341.86), with $\mathrm{I}^{2}=95.00 \%$, as shown in Fig. 2a. Most studies were located outside of the funnel in the plot, which means that the research publication bias, calculated with the Egger test, was large, as shown in Fig. 2 b.

\section{AST}

All four studies dealing with AST reported that its association with COVID -19 was statistically significant. The pooled mean for all four studies was $27.28 \mathrm{IU} / \mathrm{L}$ (95\% CI: 18.30 36.25 ), with $\mathrm{I}^{2}=87.31 \%$, as shown in Fig. 3a. Studies were located in the funnel and spread out, which means that the research publication bias was large, as shown in Fig. 3 b.

\section{ALT}

All four studies dealing with ALT reported that its association with COVID-19 was statistically significant. The pooled mean for all four studies was 24.44 IU/L (95\% CI: 15.73 33.15), with $\mathrm{I}^{2}=90.96 \%$, as shown in Fig. 4a. Most studies were located inside the funnel and spread out, which means that the research publication bias was large, as shown in Fig. $4 b$. 
Fig. 4 a Forest plot of randomeffects meta-analysis for alanine aminotransferase (ALT). b Funnel plot with pseudo 95\% confidence interval for ALT

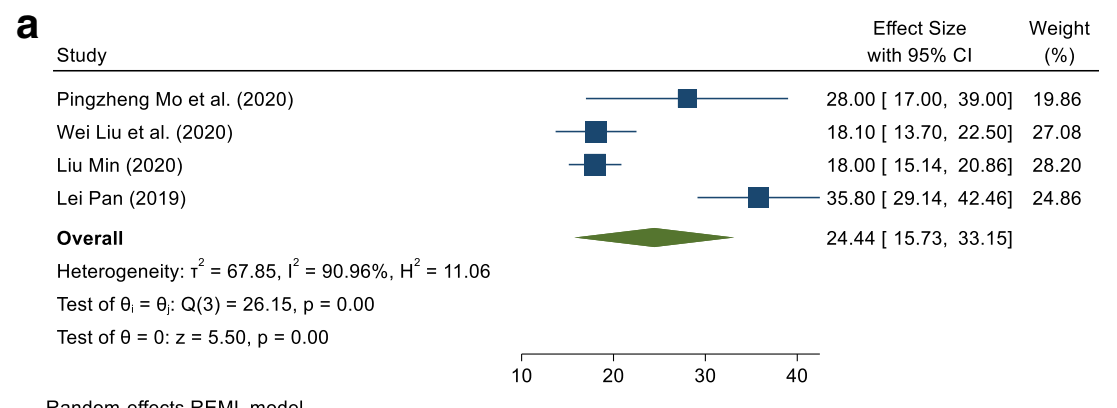

b

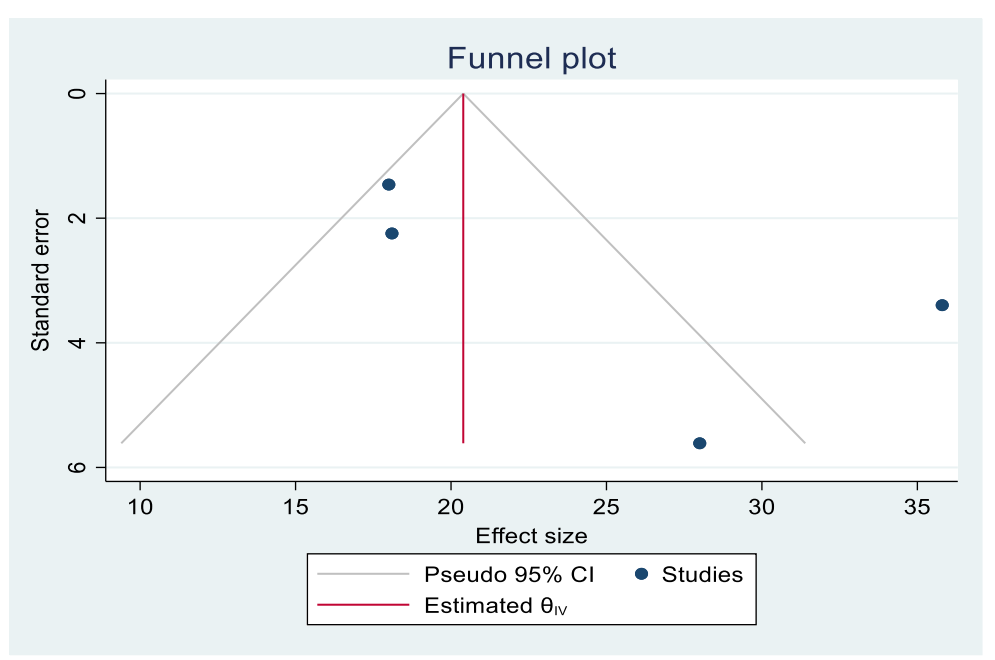

\section{Discussion}

An unknown outbreak of pneumonia occurred in Wuhan with some uncommon extrapulmonary symptoms in patients, like anorexia, diarrhea, and vomiting, not necessarily with respiratory symptoms, was caused by SARS-CoV-2, with the disease being termed COVID-19. It is spread from person to person in the community. Some patients showed lower ability to respond to treatment after hospitalization and developed severe pneumonia, pulmonary edema, ARDS, or multiple organ failure (Mo et al. 2020; Pan et al. 2020). The clinical variety of COVID-19 varies from asymptomatic or paucisymptomatic forms to respiratory failure to multiorgan and systemic manifestations in terms of sepsis, septic shock, and MODS. The symptoms in the early stages of infection were non-specific, and differential diagnosis may include a variety of infectious and non-infectious etiologies (Cascella et al. 2020).

This study used a random-effects model, with the assumption that each of the included investigations used randomly selected samples for analysis. The high heterogeneity of each variable type observed in the studies was due to the different periods in which the studies were carried out and the diversity of locations, cultures, and economic status. The cause of liver damage in COVID-19 was due to SARS-CoV-2 being similar to SARS-CoV and could invade the human body by binding to the human angiotensin-converting enzyme 2 (ACE-2) receptor, which causes liver tissue injury by upregulation of ACE-2 expression in liver tissue (Pan et al. 2020).
Table 2 Number of studies for different measures of the liver profile, with pooled mean, $95 \%$ confidence interval (CI), and measure of heterogeneity $\left(\mathrm{I}^{2}\right)$

\begin{tabular}{|c|c|c|c|c|c|c|}
\hline \multirow[t]{2}{*}{ Liver profile } & \multirow{2}{*}{$\begin{array}{l}\text { Number of } \\
\text { studies }\end{array}$} & \multirow[t]{2}{*}{ Mean } & \multicolumn{2}{|l|}{$95 \% \mathrm{CI}$} & \multirow[t]{2}{*}{$I^{2}$} & \multirow{2}{*}{$\begin{array}{l}p \text { - } \\
\text { Value }\end{array}$} \\
\hline & & & $\begin{array}{l}\text { Lower } \\
\text { limit }\end{array}$ & $\begin{array}{l}\text { Upper } \\
\text { limit }\end{array}$ & & \\
\hline Albumin & 4 & 38.84 & 35.83 & 41.86 & 95.00 & $<0.001$ \\
\hline $\begin{array}{l}\text { Aspartate aminotransferase } \\
\text { (AST) }\end{array}$ & 4 & 27.28 & 18.30 & 36.26 & 87.31 & $<0.001$ \\
\hline $\begin{array}{l}\text { Alanine aminotransferase } \\
\text { (ALT) }\end{array}$ & 4 & 24.44 & 15.73 & 33.15 & 90.96 & $<0.001$ \\
\hline
\end{tabular}


Our meta-analysis study observed that the pooled mean of albumin in COVID-19 patients was lower than the normal range, which is due to a defect in its synthesis. The AST pooled mean value was reported to be higher in COVID-19 patients, which is due to damage in hepatic tissue storage. A study suggests that, as the disease progresses, patients encounter secondary infection, which further causes abnormal liver function, due to (1) viral infection directly causing damage to liver cells and (2) drug-related liver damage (Liu et al. 2020a).

In this study, the ALT pooled mean was reported to be within the normal range. However, the AST pooled mean was not. Moreover, the albumin pooled mean was estimated as $38.84 \mathrm{mg} / \mathrm{L}$, which is statistically significant. Another study supported and found that albumin was significantly decreased in COVID-19 patients, whereas ALT and AST levels were in the normal range (Liu et al. 2020b). Compared with general patients, one study observed that COVID-19 patients had a higher level of AST and a lower level of albumin (Mo et al. 2020). Another study in China did not find any abnormalities in the liver test among COVID-19 patients (Pan et al. 2020).

\section{Conclusion}

This study has reported the pooled means for different parameters of a liver profile, which are routinely estimated to evaluate liver damage in COVID-19 patients. The pooled mean reported in this study will be useful for working out the degree of liver damage. The alanine aminotransferase (ALT) and albumin pooled means were reported to be within the normal range; however, the serum level of aspartate aminotransferase (AST) (pooled mean) was found to be high in COVID-19 patients. Also, it can be concluded from the pooled means that COVID-19 caused small alterations in liver function but not necessarily liver failure itself.

\section{Compliance with ethical standards}

\section{Conflict of interest None.}

Ethical clearance Not applicable.

Availability of supporting data This study is based on secondary data, which are available online from the sources as mentioned in the 'Materials and methods' section.

\section{References}

Cascella M, Rajnik M, Cuomo A, Dulebohn SC, Di Napoli R (2020) Features, Evaluation and Treatment Coronavirus (COVID-19). StatPearls 1-15. http://www.ncbi.nlm.nih.gov/pubmed/32150360

Jussawalla DJ, Deshpande VA (1971) Evaluation of cancer risk in tobacco chewers and smokers: an epidemiologic assessment. Cancer 28(1):244-252. https://doi.org/10.1002/1097-0142(197107)28: $1<244::$ AID-CNCR2820280150>3.0.CO;2-H

Kaplan A, Jack R, Opheim KE, Toivola B, Lyon AW (1995) Clinical chemistry: interpretation and techniques. Williams \& Wilkins, Baltimore

Liu M, He P, Liu HG, Wang XJ, Li FJ, Chen S, Lin J, Chen P, Liu JH, Li $\mathrm{CH}$ (2020a) Clinical characteristics of 30 medical workers infected with new coronavirus pneumonia. Zhonghua Jie $\mathrm{He} \mathrm{He} \mathrm{Hu} \mathrm{Xi} \mathrm{Za}$ Zhi 43(3):209-214. https://doi.org/10.3760/cma.j.issn.1001-0939. 2020.03.014

Liu W, Tao Z-W, Wang L, Yuan M-L, Liu K, Zhou L, Wei S, Deng Y, Liu J, Liu H-G, Yang M, Hu Y (2020b) Analysis of factors associated with disease outcomes in hospitalized patients with 2019 novel coronavirus disease. Chin Med J (Engl) 133:1032-1038. https://doi. org $/ 10.1097 / \mathrm{cm} 9.0000000000000775$

Mo P, Xing Y, Xiao Y, Deng L, Zhao Q, Wang H, Xiong Y, Cheng Z, Gao S, Liang K, Luo M, Chen T, Song S, Ma Z, Chen X, Zheng R, Cao Q, Wang F, Zhang Y (2020) Clinical characteristics of refractory COVID-19 pneumonia in Wuhan, China. Clin Infect Dis (in press). https://doi.org/10.1093/cid/ciaa270

Pan L, Mu M, Yang P, Sun Y, Wang R, Yan J, Li P, Hu B, Wang J, Hu C, Jin Y, Niu X, Ping R, Du Y, Li T, Xu G, Hu Q, Tu L (2020) Clinical characteristics of COVID-19 patients with digestive symptoms in Hubei, China: a descriptive, cross-sectional, multicenter study. Am J Gastroenterol 115:766-773

Sun P, Lu X, Xu C, Sun W, Pan B (2020) Understanding of COVID-19 based on current evidence. J Med Virol 92:548-551. https://doi.org/ 10.1002/jmv. 25722

Publisher's note Springer Nature remains neutral with regard to jurisdictional claims in published maps and institutional affiliations. 